\title{
Long-Term Survival in a Case of Pleomorphic Carcinoma with a Brain Metastasis
}

\author{
Keiji Yamanashi $^{\mathrm{a}}$ Satoshi Marumo ${ }^{\mathrm{a}}$ Koki Miura $^{\mathrm{b}}$ Masahiro Kawashima $^{\mathrm{b}}$ \\ ${ }^{a}$ Respiratory Disease Center, Tazuke Kofukai Foundation, Medical Research Institute, \\ Kitano Hospital, Osaka, and ${ }^{b}$ Department of Thoracic Surgery, Kishiwada City Hospital, \\ Kishiwada, Japan
}

\section{Key Words}

Lung cancer surgery $\cdot$ Adjuvant/neoadjuvant therapy $\cdot$ Metastases

\begin{abstract}
Pleomorphic carcinoma (PC) is a rare malignant lung tumor with a poorer prognosis compared to other histological types of non-small cell lung cancer. However, several recent immunohistochemical studies revealed that a low MIB-1 index is a good prognostic marker in patients with PC. We report the case of a patient with PC and a single brain metastasis that achieved long-term recurrence-free survival following treatment with combined modality therapy. In this case, the MIB-1 index was reduced by gemcitabine-based chemotherapy, which may have led to long-term disease-free survival. The MIB-1 index may be a useful biomarker for the response to chemotherapy.

(c) 2014 S. Karger AG, Basel
\end{abstract}

\section{Introduction}

Pleomorphic carcinoma (PC) is a rare malignant lung tumor characterized by dual-cell components including spindle or giant cells as well as epithelial cells. PC has been reported to have a more aggressive clinical course and a poorer survival compared to other histological types of non-small cell lung cancer (NSCLC) [1, 2]. We report the case of a patient with PC and a single brain metastasis that achieved long-term recurrence-free survival following treatment with combined modality therapy. 
Yamanashi et al.: Long-Term Survival in a Case of Pleomorphic Carcinoma with a Brain Metastasis

\section{Case Report}

A 71-year-old male presented with aphasia and muscle weakness of the left side of his body. He was suspected of having a central nervous system disorder and underwent head brain CT that showed a $50 \times 50 \mathrm{~mm}$ mass lesion in the right frontal lobe (fig. 1a). His chest Xray showed a mass shadow with a cavity in the right-middle lung field (fig. 1b). The chest CT also showed a $70 \times 68 \mathrm{~mm}$ mass with a cavity in the right lower lobe (fig. 1c). The level of tumor markers like carcinoembryonic antigen, cytokeratin 19 fragments and pro-gastrinreleasing peptide were not elevated. Based on these clinical findings, the patient was diagnosed with lung cancer with a single brain metastasis.

Craniotomy was performed because the patient's central nervous system's symptoms were severe and his Eastern Cooperative Oncology Group performance status was zero. The brain tumor in the right frontal lobe was completely removed, and the pathological diagnosis was confirmed as sarcomatoid pattern carcinoma. A CT-guided percutaneous lung biopsy proved that the pathological diagnosis of the right lung mass was spindle cell carcinoma. The patient was therefore diagnosed as having pleomorphic carcinoma of the lung with a single brain metastasis (clinical T3N0M1b stage IV).

After craniotomy, the patient underwent 6 courses of chemotherapy (carboplatin AUC 4 on day 1 and gemcitabine $800 \mathrm{mg} / \mathrm{m}^{2}$ on days 1 and 8 , repeated every 3 weeks). After chemotherapy, the chest CT showed a reduced nodular shadow $(62 \times 50 \mathrm{~mm})$ (fig. 1d), while $18 \mathrm{~F}$-fluorodeoxyglucose positron emission tomography showed no uptake in the lymph nodes (data not shown). The patient achieved a partial response by preoperative chemotherapy and underwent a right-lower lobectomy, partial resection of a right S2 segment, parietal pleurectomy, and mediastinal lymph node dissection through a posterolateral thoracotomy. This surgery was required because of the possibility of invasion into the right upper lobe and the parietal pleura.

Macroscopically, the tumor was $64 \times 48 \mathrm{~mm}$ in size (fig. 2a). Microscopically large, spindle-shaped atypical cells had sarcomatously proliferated into most of the tumor portions (fig. 2b). The tumor had invaded the parietal pleura but not the S2 segment. There was no lymph node metastasis. The final diagnosis was pathological T3N0M1b PC of the lung stage IV. Immunohistochemically, the MIB-1 index of the specimen obtained by CT-guided percutaneous lung biopsy before preoperative chemotherapy was high at $60 \%$ (fig. 2c), whereas that of the resected tumor was low at $10 \%$ (fig. 2d).

The patient is alive after 7 years, with no signs of tumor recurrence.

\section{Discussion}

PC is rare, accounting for $0.3-1.0 \%$ of all lung cancers; it is defined as a poorly differentiated NSCLC [2]. In a recent large study on patients with stage I-III lung cancer, the overall 5 -year survival rate and mean survival time of these patients were reported to be significantly lower and shorter than that of patients with other types of NSCLC (36.7 vs. 59.4\%, and 22.8 vs. 96 months, respectively) [3]. Therefore, patients with PC have a significantly poorer outcome than patients with other types of NSCLC.

In the present case, the patient underwent preoperative chemotherapy after craniotomy. He achieved a partial response by preoperative chemotherapy and underwent a right lower lobectomy. The National Comprehensive Cancer Network recommends neoadjuvant chemotherapy combined with surgical treatment or surgical treatment combined with adjuvant chemotherapy in NSCLC cases with brain metastasis (TNM category T1-2, N0-1, 
T3N0 if the metastasis can be controlled). Although previous papers showed that PC had a poor response to chemotherapy regimens [4], there is a report indicating the efficacy of chemotherapy for PC [1]. In our case, gemcitabine-based chemotherapy was effective and suggested that this treatment was potentially useful for PC.

The following discussion includes the possible reasons why the present case achieved long-term survival. Fishback et al. [2] showed the following 4 factors that contributed to the poor prognosis for a PC survival: (1) tumor size $>5 \mathrm{~cm}$, (2) stage greater than I at presentation, (3) presence of distant metastases, and (4) lymph node metastases. In this case, the absence of lymph node metastasis was a good prognostic factor. These 4 factors only include the TNM classifications and are common prognostic factors for PC and other NSCLCs. However, patients with PC have poorer outcomes than those with other NSCLCs, and it is therefore necessary to investigate specific prognostic factors for PC.

In the present case, the MIB-1 index of the immunohistochemical specimen obtained by CT-guided percutaneous lung biopsy before preoperative chemotherapy was high at $60 \%$ (fig. 2c), although it was reduced to $10 \%$ in the resected tumor (fig. $2 \mathrm{~d}$ ). MIB-1 is present during all active phases of the cell cycle (G1, S, G2, and mitosis), but absent in resting cells (G0). This property makes it an excellent marker for determining the so-called growth fraction of a given cell population [5]. In patients with PC, a low MIB-1 index $(<35 \%)$ is reported to be a good prognostic marker [6]. Moreover, there is a report in which the MIB-1 index was significantly lower after gemcitabine-based chemotherapy and exerted a true antiproliferative effect on tumors in vivo. Reduced proliferation with gemcitabine-based chemotherapy may partly be because of increased apoptosis in actively proliferating cells so that the residual population would be enriched by MIB-1-negative cells [7]. In our case, the MIB-1 index was reduced by gemcitabine-based chemotherapy, which may have led to longterm disease-free survival. The MIB-1 index may therefore be a useful biomarker of the response to chemotherapy, with the reduction in the index following preoperative chemotherapy representing a response marker that reflects the proliferation of tumor cells. The changes in the MIB-1 index caused by preoperative chemotherapy may therefore provide useful information for deciding the regimen for adjuvant chemotherapy.

\section{Disclosure Statement}

The authors declare that they have no conflicts of interest.

\section{References}

$>1$ Chang YL, Lee YC, Shih JY, Wu CT: Pulmonary pleomorphic (spindle) cell carcinoma: peculiar clinicopathologic manifestations different from ordinary non-small cell carcinoma. Lung Cancer 2001;34:91-97.

-2 Fishback NF, Travis WD, Moran CA, Guinee DG Jr, McCarthy WF, Koss MN: Pleomorphic (spindle/giant cell) carcinoma of the lung. A clinicopathologic correlation of 78 cases. Cancer 1994;73:2936-2945.

3 Mochizuki T, Ishii G, Nagai K, et al: Pleomorphic carcinoma of the lung: clinicopathologic characteristics of 70 cases. Am J Surg Pathol 2008;32:1727-1735.

4 Ito K, Oizumi S, Fukumoto S, et al: Clinical characteristics of pleomorphic carcinoma of the lung. Lung Cancer 2010;68:204-210.

5 Scholzen T, Gerdes J: The Ki-67 protein: from the known and the unknown. J Cell Physiol 2000;182:311322.

6 Pelosi G, Fraggetta F, Nappi O, et al: Pleomorphic carcinomas of the lung show a selective distribution of gene products involved in cell differentiation, cell cycle control, tumor growth, and tumor cell motility: a clinicopathologic and immunohistochemical study of 31 cases. Am J Surg Pathol 2003;27:1203-1215. 
Yamanashi et al.: Long-Term Survival in a Case of Pleomorphic Carcinoma with a Brain Metastasis

7 Sanchez-Rovira P, Anton A, Barnadas A, et al: Classical markers like ER and ki-67, but also survivin and $\mathrm{pERK}$, could be involved in the pathological response to gemcitabine, adriamycin and paclitaxel (GAT) in locally advanced breast cancer patients: results from the GEICAM/2002-01 phase II study. Clin Transl Oncol 2012;14:430-436.
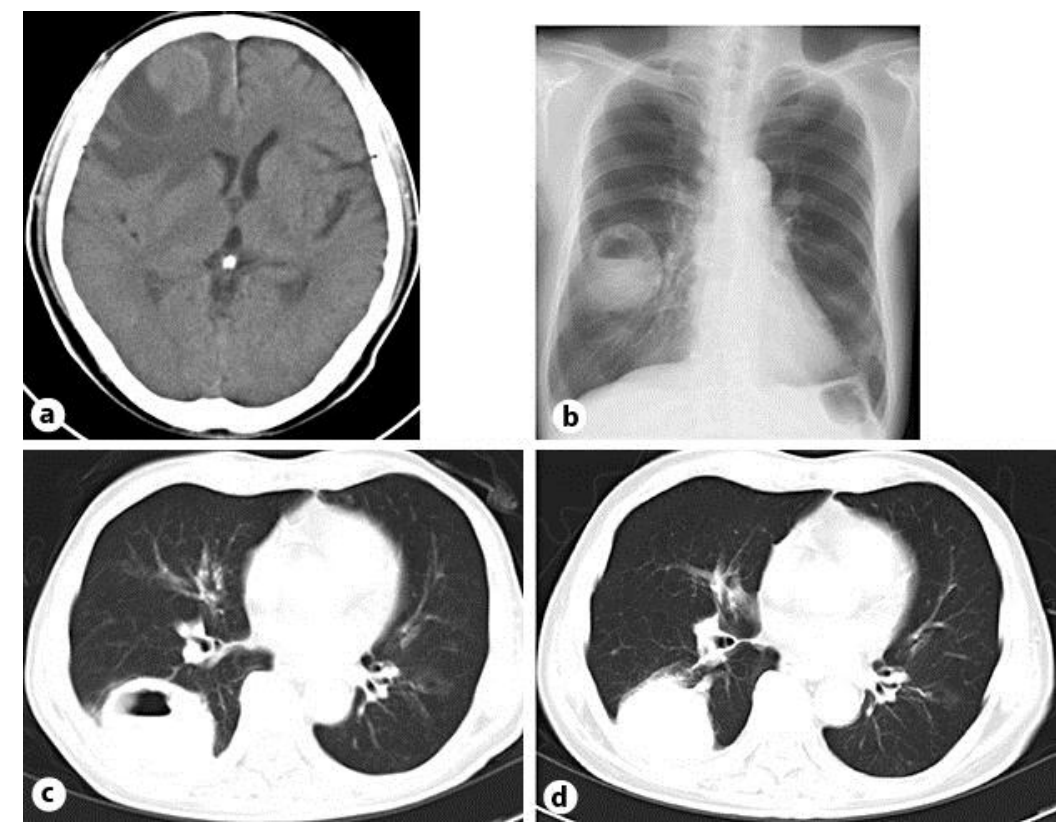

Fig. 1. a Brain CT showing a $50 \times 50 \mathrm{~mm}$ mass lesion in the right frontal lobe. b Chest X-ray showing a nodular mass shadow with a cavity in the right-middle lung field. c, $\mathbf{d}$ Chest CT showing a $70 \times 68 \mathrm{~mm}$ mass with a cavity in the right lower lobe (c) and a reduced nodular shadow $(62 \times 50 \mathrm{~mm})$ after 6 courses of chemotherapy (d). 


\section{Case Reports in Oncology}

\begin{tabular}{l|l}
\hline Case Rep Oncol 2014;7:799-803 \\
\hline DOI: $10.1159 / 000368186$ & $\begin{array}{l}\text { C 2014 S. Karger AG, Basel } \\
\text { www.karger.com/cro }\end{array}$ \\
\hline
\end{tabular}

Yamanashi et al.: Long-Term Survival in a Case of Pleomorphic Carcinoma with a Brain Metastasis
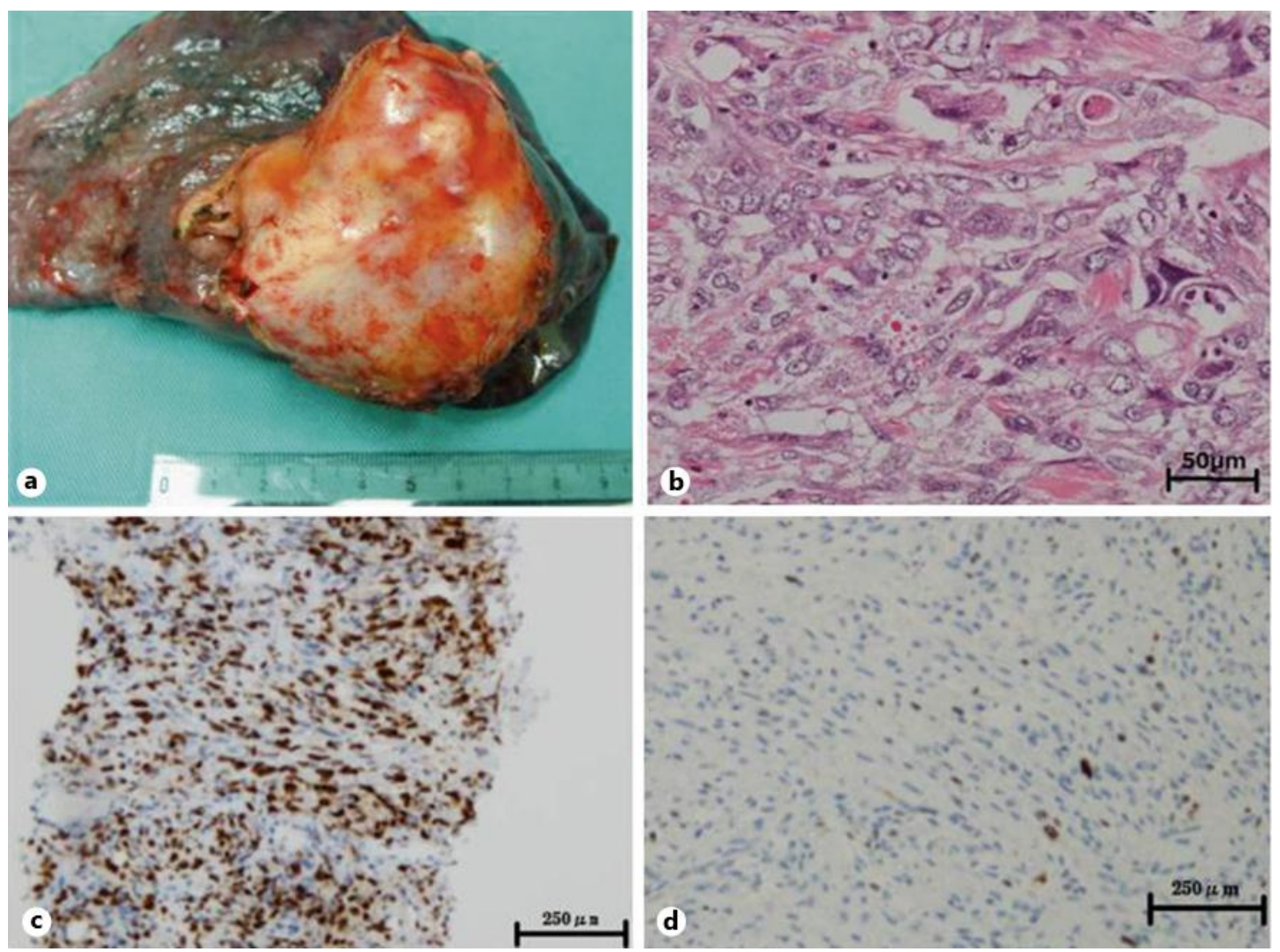

Fig. 2. Postoperative specimen of the lung showing a $64 \times 48 \mathrm{~mm}$ tumor in the right lower lobe macroscopically (a) and large, spindle-shaped atypical cells proliferating sarcomatously into most of the tumor portions microscopically (b). The MIB-1 index in the specimen obtained by CT-guided percutaneous lung biopsy before preoperative chemotherapy is high at $60 \%$ (c), but low at $10 \%$ in the specimen of the resected tumor $(\mathbf{d})$. 